\title{
La danse du masque noir. Rites funéraires des Mossé du Kadiogo (Burkina Faso)
}

Jean-Marc Durantel

\section{(2) OpenEdition}

1 Journals

Édition électronique

URL : http://journals.openedition.org/span/1430

DOI : 10.4000/span. 1430

ISSN : 2268-1558

Éditeur

École pratique des hautes études. Sciences humaines

\section{Édition imprimée}

Date de publication : 1 décembre 1994

Pagination : 219-229

ISSN : 0294-7080

\section{Référence électronique}

Jean-Marc Durantel, « La danse du masque noir. Rites funéraires des Mossé du Kadiogo (Burkina

Faso) », Systèmes de pensée en Afrique noire [En ligne], 13 | 1994, mis en ligne le 17 février 2014, consulté le 19 avril 2019. URL : http://journals.openedition.org/span/1430 ; DOI : 10.4000/span.1430

Ce document a été généré automatiquement le 19 avril 2019

(c) École pratique des hautes études 


\title{
La danse du masque noir. Rites funéraires des Mossé du Kadiogo (Burkina Faso)
}

\author{
Jean-Marc Durantel
}

1 Les masques dont nous allons étudier l'intervention au cours des rites funéraires sont appelés en moré wan-sablassé, c'est-à-dire " Masques noirs ", à cause de la longue « robe » de fibres végétales teintes en noir qui enveloppe et dissimule totalement le corps du danseur. Notre observation a porté sur un groupe d'une dizaine de villages, dans la région de Ziniaré, à quelques soixante kilomètres de Ouagadougou. Le wan-sablassé, chez les Mossé, est nécessairement sacré. Il ne s'exhibe ni « sur la place du marché », ni en aucun autre endroit ou occasion «où l'homme rit». Seuls la mort et le deuil justifient son intervention.

2 Le décès d'un homme donne lieu à deux cérémonies distinctes et qui peuvent être très éloignées l'une de l'autre dans le temps : l'enterrement d'une part, les « funérailles » (en moré : kouré) d'autre part, qui doivent permettre à l'âme du défunt de rompre ses liens avec le monde des vivants et de rejoindre ses ancêtres. Chacune de ces cérémonies se clôt par une intervention du masque qui reproduit, en les mimant, les étapes de ces cérémonies. Ce " jeu », au sens théâtral du terme, du masque est appelé suku-ce que l'on peut traduire par « danse du masque noir».

3 Enterrement et funérailles donnent chacun lieu à un suku particulier; dans le premier cas, il s'agit du rag-nor suku, «danse du masque noir devant la porte de la case » (sousentendu : du mort); dans le second cas, il s'agit du wé-keeng suku, « danse du masque noir dans la brousse lointaine $"$.

Le masque noir s'exprime sur plusieurs modes. Il fait intervenir une langue secrète, le sankandé, réservée au suku et dont l'emploi hors de ce contexte est rigoureusement prohibé, un langage « musical » - tambouriné -, et enfin un langage gestuel - une danse propre à chaque masque. En fait, le suku suppose rien moins que les règles d'un «théâtre sacré ", avec une scène où des acteurs venus de l'Au-delà jouent - miment - les actes de la 
vie des hommes. Cette expression scénique suppose l'intervention simultanée de trois acteurs au moins, dont aucun n'utilise le même langage : 1) le masque (langage gestuel) ; 2) le wilma ou su-goamda, orateur qui s'adresse au masque au nom du chef des masques en utilisant la langue secrète, sankandé; 3) les tam-tams (langage tambouriné).

\section{Le rag-nor suku}

5 Le rag-nor suku intervient pour achever la cérémonie publique de l'enterrement. Le corps du défunt a été enterré, et il est donc absent de la scène. Cette "danse du masque noir » constitue une reprise mimique, théâtrale, de la cérémonie d'inhumation proprement dite qui l'a précédée.

6 Selon la coutume, ce rituel comporte 333 mouvements et séquences - ce chiffre apparaissant chez les Mossé comme le symbole par excellence de l'Au-delà - mouvements et séquences qui vont des civilités du masque envers les responsables visibles et invisibles de la localité lors de son arrivée, jusqu'aux rites qui accompagnent son départ, en passant par les multiples jeux scéniques mimant les raisons de sa venue parmi les hommes, la recherche du cadavre, sa découverte, ainsi que les phases successives du rite d'enterrement - précédées par le « récit » de l'origine du décès : la maladie du vieux, les soins prodigués en vain, les consultations d'un devin, etc.

7 Les masques sont ici des esprits surnaturels venus du «Ciel»: à leur tête se trouve un " chef de délégation », un masque "supérieur » que l'on nomme lui aussi kouré (du terme qui désigne les « funérailles ») mais qui n'est pas le « chef suprême » des masques, celui-ci restant au village des masques auprès des fétiches, implorant qu'il n'arrive aucun malheur aux masques en déplacement. C'est ce masque, l'aîné des masques en quelque sorte, qui ouvre le suku. C'est également à lui que revient le devoir de saluer la terre du village où va se dérouler le rituel, lors de l'arrivée des masques.

C'est par le message introductif suivant que le masque kouré salue ses hôtes:

«À ceux qui gouvernent la Terre, nos respects !

Aux horizons de cette terre, nos respects !

Aux Génies et aux Morts de cette terre, nos respects !

Aux pierres angulaires sacrées de cette terre, nos respects!

Nous sommes venus sur cette terre, en portant l'amitié dans notre cœur

Et nous ne pouvons exprimer la Parole et le Geste,

Sans au préalable rendre le respect à ce que cette terre respecte. »

Le masque ne parle pas, c'est le su-goamda (l'orateur, celui qui dialogue avec le masque et le dirige pendant son intervention) qui s'exprime en son nom, dans la langue secrète. Ces salutations accomplies, les masques (sauf le kouré) se rendent dans un enclos en séco (natte de paille) appelé kargo situé à quelques deux ou trois cents mètres de la concession du défunt, tandis que le su-goamda s'installe à une centaine de mètres de là, assis sur un mortier retourné, à côté des joueurs de tam-tams et de flûtes; en un troisième lieu, un peu à l'écart, formant un triangle avec le groupe des masques et celui de l'orateur et des musiciens, également à l'abri d'un enclos de séco, est gardé un fétiche protecteur des masques, le tiibo, sur lequel on sacrifie un poulet et une chèvre avant que ne débute le suku.

Pendant toute la cérémonie, c'est le su-guomda qui « mène la danse » : il donne des ordres au masque, le questionne, aborde avec lui des aspects de la spiritualité ou lui commande d'accomplir les souhaits de la communauté. Les réponses du masque se manifestent par le 
mouvement: s'il frissonne ou remue la tête, la réponse est négative; les autres gestes expressifs sont interprétés comme des réponses positives. Le masque possède également un sifflet et un fouet, symboles du caractère divin de son expression; si on lui demande de fouetter le sol ou la case mortuaire, cela signifie, contrairement à l'usage humain, qu'on lui demande de donner sa bénédiction ou de présenter ses respects au sol (aux génies, aux morts) ou à la personne défunte.

11 Le rituel du rag-nor suku débute par l'invocation suivante, proférée par le su-goamda, qui appelle le masque de la façon suivante :

«Il est une parole.

J'ai appris que l'Etranger est venu sur cette terre.

Mais son visage m'est encore inconnu.

Il est une Parole.

Je voudrais voir l'Esprit masqué.

Il est une Parole, viens, ton père est absent.

Viens avec le fouet, frappe la maison de la Mort.

L'étranger est venu sur cette terre,

Mais son visage m'est encore inconnu.

J'ai vu son visage. Son visage est beau.

Je voudrais voir son côté droit. Son côté droit est beau.

(...)

Dieu est au-dessus de nous. Mais c'est Dieu qui connaît le sens de ta venue.

Rends hommage à Dieu.

Dieu a reçu son fouet.

Mais rends hommage à Tenga son épouse ;

(...)

Aux génies et Fétiches du village ;

Rends hommage au responsable du village. »

Après cette partie introductive, l'orateur introduit ce qui va suivre de la manière suivante :

" ${ }^{\circ} \mathrm{Tu}$ es venu, je t'ai vu, mais j'ignore les raisons de ta venue. Tu as quitté le Ciel

pour venir sur la terre ;

Est-ce la puissance de la Parole qui t'a amené sur la terre?

Exprime-toi que je comprenne. »

13 Suit toute une série de questions visant à connaître les raisons de la venue du masque : at-il quitté le «Ciel » par manque de nourriture, de boisson, de femme, d'amis? A chaque fois le masque répond par la négative. Puis il exécute des mouvements vers le Ciel, vers la terre, se met à languir et finit par aller se prostrer contre la case du mort. L'orateur comprend et reprend ainsi :

«J'ai compris ton message.

Tu as dit : si je suis venu, ce n'est pas pour un marché,

Je ne suis pas là pour tout ce que tu as dit ;

Je ne vais jamais au puits, je ne vais jamais là où l'homme peut rire ;

Je ne suis que là où l'homme est mort. Le vieux de la maison est malade,

C'est pour cela que j'ai quitté le Ciel pour venir sur la terre,

Et pleurer avec les hommes.

J'ai compris ton message.

L'homme de la case est malade.

Cherche son argent, va chez le devin,

Implore Dieu pour lui obtenir la santé. »

14 Le masque se lève alors, se dirige vers la case mortuaire, fait mine de réfléchir et de chercher quelque chose, puis il revient vers l'orateur en dansant. L'orateur traduit : 
«J'ai compris ton message.

Le devin a dit de chercher de l'eau de farine, de chercher un poulet, d'aller dans la brousse, d'offrir aux Ancêtres,

Pour qu'ils implorent Dieu et obtiennent la santé. »

Le masque acquiesce, puis se dirige vers la case mortuaire, se prosterne comme pour chercher quelque chose, puis s'éloigne et s'accroupit comme pour un sacrifice; après quelques instants, il se relève péniblement, hochant doucement la tête, et revient vers le su-goamda. Celui-ci expose le sens de ce mime de cette façon :

" ${ }^{\circ} \mathrm{J}$ 'ai compris ton message.

Tu as dit que la force du devin est impuissante ;

Que la force des Ancêtres est impuissante ;

Que le souffle de l'ancien de la maison est à son terme et qu'il s'éteint.

Cherche un poulet, entre dans la case ;

Demande à l'ancien la cause de sa mort.

Est-ce un vœu de lui-même, ou est-ce le fait d'un homme?»

de la façon suivante: de part et d'autre du cadavre sont disposés un plat de sagbo (plat traditionnel à base de mil) et un plat de sauce ; tour à tour, chaque membre de la famille enlève une poignée de sagbo, enjambe le corps, trempe la nourriture dans la sauce et avale en prononçant une prière pour le mort. L'orateur poursuit :

«Si le mort mange le repas, du sentier où le retour est impossible,

Le mort ne doit plus rester couché en ce lieu.

Il faut donc le faire sortir. »

«J'ai compris ton message.

Tu as dit que le vieux de la maison a dit qu'il était à la fin de ses jours,

Et il a répondu à l'appel de ses Pères.

Que Dieu apaise la terre pour qu'il se repose. »

« La parole est épuisée, mais il reste encore un peu.

Va chercher une pioche, une houe, le plat en bois,

Eloigne-toi des maisons, cherche un emplacement pour la tombe.

Va dans la brousse, ramène du som-piga (graminée sauvage),ramène une feuille de

karité, ramène une épine,

Pose le tout derrière la maison (en moré : $N$ degl tampura ${ }^{1}$, zug),

Cherche de l'eau, pose au même lieu, pour qu'on fasse la toilette du mort,

Amène un rasoir, pour qu'on coiffe le mort et qu'il soit beau. »

réunit toute la famille autour du défunt, avant l'inhumation :

« Retourne dans la maison, fais préparer le dernier repas sacré ;

Fais préparer la dernière sauce sacrée, pour la nourriture du mort.

Il a faim. Son voyage sera long.

Et qu'on le prenne pour la case de son repos. »

Le masque va " exécuter » - par le mime - ces instructions du su-goamda. Il retourne donc à la case, prend un peu de temps, puisqu'il est censé laver et habiller le mort, puis il sort en donnant l'impression qu'il enlève le séco qui ferme la porte et le pose sur le sol ; on le voit ensuite faire sortir le mort qu'il couche sur la natte. Enfin le masque s'immobilise 
dans la direction supposée des pieds du cadavre; c'est ce dernier geste, hautement significatif, que le su-goamda va ensuite commenter :

«L'âné des orphelins a dit que son père est mort.

Il apporte un poulet : prend et offre à son père.

C'est le « poulet du pied».

Pour qu'il atteigne son père sous terre,

Ses ancêtres sous terre,

Et qu'il lui apporte la santé. »

Il s'agit ici d'une référence à un rite que l'on pourrait appeler « rite d'ouverture des yeux du mort ». Il faut savoir que chez les Mossé, le monde de l'Au-delà est caractérisé par une inversion systématique des éléments de l'Ici-bas ; ainsi, en particulier, lorsqu'un homme meurt, sa vision se trouve transférée du sommet du corps vers sa base, c'est-à-dire de la tête dans les pieds. Mais encore faut-il procéder au rite approprié, lequel consiste en un sacrifice de poulet sur les pieds du mort. Le sang qui coule sur les orteils ouvre les « yeux des pieds ». C'est après ce sacrifice que l'on peut procéder à l'interrogatoire rituel du cadavre si la mort est suspecte. Sinon, l'orateur poursuit ainsi son allocution :

«Les anciens du village ont dit que son absence est une douleur pour eux;

Ils te remettent cet animal à son intention,

En signe de respect et de soumission aux ancêtres sous terre. »

L'orateur peut alors passer en revue, à la suite des anciens, tous les « corps sociaux », les autorités, puis tel ou tel individu, avant de reprendre :

" ${ }^{\circ}$ Prend, prend le mort.

Va avec lui dans la case où il n'est pas de retour. Va avec lui.

S'il part le jour, il ne reviendra pas la nuit.

S'il part la nuit, il ne reviendra pas le jour. »

Le masque se penche alors, comme pour prendre le corps, puis se dirige vers la tombe où il s'arrête quelque temps, dans une attitude de méditation; enfin, gravement, il revient vers l'orateur, comme s'il avait procédé à l'inhumation. La cérémonie touche alors à sa fin ; le su-goamda achève ainsi son allocution :

«Va! Entre dans la maison du défunt; cherche l'eau ;

Sors! Offre à la population, à tous ceux qui sont debout.

Nos condoléances d'hier, nos condoléances d'avant-hier, nos condoléances du

passé.

La parole est terminée ;

C'est Dieu qui remerciera, c'est Dieu qui vous la rendra à notre place. »

Pendant ce temps, le masque frappe le sol de son fouet, saluant ainsi l'assistance, tandis que l'orateur le congédie ainsi :

«Rentre à la maison pour prendre du repos

Car ta tête te fait mal, tes pieds te font mal. »

Le masque quitte alors l'aire de « danse » et retourne, toujours accompagné d'un enfant, jusqu'au kargo (l'enclos des masques) où il rejoint les autres masques, qui vont à leur tour, un à un, s'exprimer.

Ainsi s'achève donc le rag-nor suku, qui consiste, comme nous l'avons vu, en un véritable mime, en une reconstitution théâtrale, jouée par le masque, esprit de l'Au-delà, de la cérémonie de l'inhumation qui l'a précédée. Ce rite ne concerne toutefois que le corps de l'homme : il s'agit ensuite de s'occuper de son «âme vagabonde », pour lui permettre de parvenir jusqu'aux ancêtres. C'est là le but des "funérailles", au cours desquelles se déroule le wé-keeng suku. 


\section{Le wé-keeng suku} concernent que les personnes âgées ou ayant occupé des fonctions publiques importantes dans la société. Il n'est pas nécessaire de faire intervenir les masques pour des enfants morts en bas âge ou pour une personne morte jeune et sans responsabilité collective ; par ailleurs, sont exclues des funérailles toutes les personnes mortes de mort violente, « non naturelle », telles que l'accident, le meurtre, le suicide... Il s'agit donc ici de procéder à la rupture définitive du défunt avec le monde des vivants, c'est-à-dire de permettre à l'esprit (kiima) errant du mort de quitter la terre et d'entreprendre le voyage qui le mènera jusqu'aux ancêtres; de la qualité du kouré dépendra en partie l'accueil que ses Pères réserveront au défunt. suku. Ils commencent par se rassembler devant la tombe du mort et, par ordre d'ancienneté, chacun va frapper de son fouet la tombe, ce qui est la manière de saluer propre au masque ; puis les masques se rassemblent dans le kargo situé, théoriquement, en brousse, à la limite du village, d'où le nom de ce rite, qui vient de weogo, la brousse. Le masque kouré assiste seul, pour éloigner les esprits malfaisants, au remplissage des canaris de raam (bière de mil) qui serviront pendant la cérémonie. Il apporte ensuite une calebasse de bière de mil (en réalité, ce n'est pas le masque qui porte, mais un adolescent qui l'accompagne) au su-goamda (l'orateur); celui-ci, assis sur un mortier retourné et tenant à la main son "bâton de cérémonie " (un bâton fourchu le long duquel sont attachés des morceaux de calebasse), prend la calebasse et la remet au tambourinaire qui en verse tout le contenu à terre. Alors les tambours se mettent en action; le su-goamda débute ainsi son allocution, en appelant et en saluant le premier des masques :

« Maître de la brousse lointaine (wé-keeng naaba),

Je vous adresse mes condoléances!

Mes condoléances d'hier, mes condoléances d'avant-hier, mes condoléances des

temps passés.

Des Paroles Profondes vont commencer. »

Le wé-keeng suku n'a pas le caractère grave du rag-nor suku. Les masques sortent un à un du kargo (l'enclos des masques) pour venir s'exprimer sur l'aire de «danse » devant le sugoamda, exécutant ses ordres ou entamant un dialogue avec lui, chacun suivant son mode d'expression propre. Le discours de l'orateur, ainsi que l'expression gestuelle du masque, porte la plupart du temps sur des paraboles, soit que la « Parole Profonde » soit proférée par l'orateur et dans ce cas le masque la retranscrit par le mouvement, soit que le masque lance un thème que l'orateur reprend. Les Paroles Profondes peuvent porter sur l'origine du monde, des hommes, d'une institution ou d'une catégorie sociale. Elles peuvent aussi exprimer une intention morale, par exemple :

«Un vieil os dans un champ de maïs ;

Les fourmis s'associent par centaines, mais ne peuvent le déplacer ;

Un chien voleur survient et l'emporte chez lui. »

31 Ce qui signifie que, dans la vie, le nombre, la volonté, le bon droit ne suffisent pas toujours, la force aveugle, la bêtise peuvent l'emporter sur tout cela.

Ainsi, la "littérature » du wé-keeng suku est essentiellement composée de paraboles morales, de récits historiques ou mythiques, de relations de faits sociaux ou de 
descriptions d'actes de la vie quotidienne. Le su-goamda peut encore, par exemple, poser au masque la question suivante :

« Nous sommes en saison pluvieuse ; il a plu le matin; que peux-tu constater le soir

et la nuit?»

masque, par des mouvements, décrit le soleil levant, le ciel qui s'assombrit, la pluie et la fin de la pluie, puis les travaux des champs de l'après-midi ; à une certaine position du soleil, qu'il représente, il montre les femmes quittant les champs pour aller ramasser l'herbe sèche en brousse. La nuit tombée, il décrit les femmes se rendant dans la brousse, s'installant près d'une termitière, allumant un feu et capturant les termites qui sortent.

On peut affirmer que, lors du wé-keeng suku, toute l'assistance est, non pas spectatrice, mais actrice, seuls étant spectateurs Dieu et les ancêtres. Ainsi, des membres de la communauté peuvent entrer en scène et danser; il faut préciser que tous ceux qui pénètrent ensemble sur l'aire de danse doivent appartenir à la même classe d'âge, c'est-àdire, en fait, à la même classe initiatique.

Ainsi se manifeste le caractère beaucoup plus joyeux et « désinvolte » du wé-keeng suku par rapport au rag-nor suku. On peut en effet y voir les masques eux-mêmes se livrer à des facéties, chacun allant jusqu'à caricaturer le comportement de l'animal dont il est l'effigie: acrobaties du masque-buffle, pitreries du masque-singe, raideur et fierté du masque-coq, coquetteries du masque-demoiselle...

Par ailleurs, ces funérailles se doivent d'être les plus somptueuses possibles car le kiima du défunt apparaît en quelque sorte comme un ambassadeur de la communauté auprès des ancêtres, qui doit précisément donner de cette communauté la meilleure image possible; c'est "riche» et auréolé de funérailles magnifiques que le défunt doit apparaitre aux yeux de ses Pères. C'est parfois la raison pour laquelle un défunt peut attendre si longtemps ses funérailles, car il faut du temps à la famille pour rassembler les richesses nécessaires.

\section{NOTES}

1. Le tampuré est un lieu situé à une dizaine de mètres derrière la maison et où l'on jette les ordures : c'est là que généralement on fait la toilette du mort. 
INDEX

Population Mossé

Mots-clés : funérailles, masques, fibre, danse, sacrifice, pieds, tête

Keywords : funerals, masks, fiber, dance, sacrifice, feet, head

Index géographique : Burkina Faso 